\title{
Thermal Damage in Diamonds: the Protective Effect of Titanium Coating
}

\author{
Marcio Carneiro Garcia Rosa ${ }^{a}$, Matheus Paes Peçanha ${ }^{a}$, Marcello Filgueira ${ }^{a *}$ \\ aPrograma de Pós-Graduação em Engenharia e Ciência dos Materiais - PPGECM, \\ Centro de Ciência e Tecnologia - CCT, Universidade Estadual do Norte Fluminense - UENF, \\ Av. Alberto Lamego, 2000, CEP 28013-602, Campos dos Goytacazes, RJ, Brazil
}

Received: May 30, 2014; Revised: July 3, 2015

\begin{abstract}
Many of the applications of diamonds expose them to high temperatures and atmospheres containing oxygen. Under these circumstances two important phenomena responsible for the thermal damage of diamonds are: oxidation and graphitization. It is well known that coatings on diamonds provide a better performance, but the amount of information in literature about the protective mechanism of these coatings is very scarce. The present work proposes a study about the thermal damage effect on diamonds with titanium (Ti) coating comparing with diamonds without it. Samples of uncoated diamonds and Ti- coated diamonds were heat treated at $1200{ }^{\circ} \mathrm{C}$ during different periods of time and characterized prior to and after the treatments. Differential thermal analysis (DTA) was performed to observe the temperatures that transformations in diamonds occur. The analysis of phases present in the samples and the observation of new phases formed were carried out by means of X-ray diffraction (XRD). In order to observe the diamond surface and how the chemical elements are distributed in it, the samples were analyzed by scanning electron microscopy (SEM) and energy-dispersive X-ray spectroscopy (EDS). The results prove the protective capability of the Ti coatings, clarifying the protective mechanism against the thermal damage.
\end{abstract}

Keywords: thermal damage, titanium coating, oxidation, graphitization

\section{Introduction}

The advent of synthetic diamonds has made it possible to obtain grains with different mechanical and physical properties, modifying only the synthesis process. It allows a wide range of applications, such as metalworking, ceramic polishing or the cut of high-hardness granite stones ${ }^{1,2}$.

Most of the applications of diamonds in industry involve exposure to high temperatures and oxidizing atmospheres ${ }^{3-5}$. This environment provides conditions for two competitive events: oxidation and graphitization. These mechanisms can occur during the synthesis of diamonds, processing of diamond tools, as well as in their use.

The oxidation of diamonds is the reaction of oxygen with carbon yielding the gaseous products $\mathrm{CO}$ e $\mathrm{CO}_{2}^{[6]}$. This phenomenon causes changes in several properties, such as: chemical reactivity, electrical conductivity, thermal conductivity and compressive strength ${ }^{7}$. According to Lee et al. ${ }^{8}$, the oxidation of diamonds in high temperatures is harmful to their applications.

According to Sun et al. ${ }^{9}$, graphitization is a critical problem to the usage of synthetic diamonds in industry, because graphite and diamonds have different properties, compromising its application in industry.

It is known that the graphitization does not take place on the entire surface of the diamond, but it starts on small triangular sites that propagate throughout its surface ${ }^{10-12}$. The graphitization occurs preferentially on surfaces $\{111\}$ and $\{110\}$, showing lower rates on surfaces $\{100\}^{13}$.

*e-mail: marcello@uenf.br
According to Evans \& Phaal $^{10}$, in order to study the graphitization one should be careful that oxidation does not occur, since their studies show that through oxidation, layers of carbon can be formed on the surface of diamonds.

It is known that the use of coatings on diamond crystals contributes positively when used in diamond tools, but the understanding of the mechanisms of oxidation and graphitization in coated diamonds were scarcely found in the literature ${ }^{2,4,5,11,12}$.

In this work, the effect of a titanium (Ti) coating as protective barrier against oxidation and graphitization of diamonds has been investigated.

\section{Experimental Procedure}

For the study of the protective effect of titanium coating, samples of cube-octahedral uncoated diamonds and Ti-coated diamonds were employed. Both diamonds are from Element Six with an average crystal size of $400 \mu \mathrm{m}$.

These samples were heat treated in a tubular resistive furnace (bp engenharia) at $1200{ }^{\circ} \mathrm{C}$ in air along different periods: 60, 180 and 360 minutes. The characterization of the samples by thermal analysis was carried out prior to heat treatments. Other characterization methods utilized were carried out prior to and after heat treatments.

In order to evaluate the behavior of diamonds at high temperatures, due to possible events of oxidation and graphitization, differential thermal analysis (DTA) was 
performed. It was applied heating rate of $5^{\circ} \mathrm{C} / \mathrm{min}$ in the thermal analysis system from TA Instruments.

X-ray diffraction (XRD) was realized to identify the phases present in the samples without heat treatment and the formation of new phases after the treatments, such as oxides and graphite. This technique was performed in a diffractometer from Shimadzu, model PDA-7000, with the following parameters: $2 \theta$ varying from 5 to $120^{\circ}$, radiation $\mathrm{CuK} \alpha$, speed $0.02^{\circ} / \mathrm{sec}$. So as to identify components present in the samples, the obtained results were compared with JCPDS (Joint Committee of Powder Diffraction Standards) charts.

The microstructure of the samples was analyzed by means of scanning electron microscopy (SEM). With this technique, it was possible to obtain topographical images of the examined surface, as well as to provide details from the surface. It was made use of an electron microscope (SEM) from Shimadzu, model SSX-550, operated at different magnifications in secondary electron mode, a $15 \mathrm{kV}$.

Qualitative analysis was carried out by means of energy-dispersive X-ray spectroscopy (EDS) in order to obtain information about the distribution of chemical elements of the samples by the mapping of characteristic $\mathrm{X}$-rays. The spectrometer is coupled to the SEM.

\section{Results and Discussion}

\subsection{Thermal analysis}

Both uncoated and Ti-coated diamonds were analyzed by means of DTA. Figure 1 shows the results for uncoated diamonds.

Through the DTA curve, it can be noted a thermal event around $900{ }^{\circ} \mathrm{C}$ that can be attributed to the phenomenon of graphitization. This result attests the temperature used as sufficient to the observation of the phenomena of thermal damage in diamonds. Rosa ${ }^{11}$ and Chagas ${ }^{12}$ reported that $1200{ }^{\circ} \mathrm{C}$ is the average temperature utilized in the processing of monocrystalline diamonds, diamond cutting tools, as well as when they are subjected to industrial use.

Figure 2 shows the results obtained by means of thermal analysis for Ti-coated diamonds. A thermal event is observed at $912.40{ }^{\circ} \mathrm{C}$ and other close to $680{ }^{\circ} \mathrm{C}$. It will be further shown that this lower temperature event is related to the oxidation of the Ti layer.

\subsection{Structural and microstructural analysis}

\subsubsection{Prior to heat treatment}

The diffractogram of the uncoated diamonds and with Ti coating is shown in Figure 3. It can be observed that both diffractograms show little noisy backgrounds and considerable intensities. In Figure 3a, the three peaks are characteristic from diamond. For diamonds with Ti coating (Figure $3 \mathrm{~b}$ ), the peaks with angle $2 \theta$ of $35.82^{\circ}, 38.44^{\circ}$ and $40.10^{\circ}$ are inherent to metallic $\mathrm{Ti}$ and the peak with angle $2 \theta$ of $90.98^{\circ}$ is inherent to titanium oxide ( $\mathrm{TiO})$.

Observing Figure 4, it is noted that both uncoated diamonds and Ti-coated diamonds do not show defects, wear, oxidation and graphitization, prior to heat treatment.

Analyzing the mapping by EDS in Figure 5, the majority composition of carbon was verified for uncoated diamonds

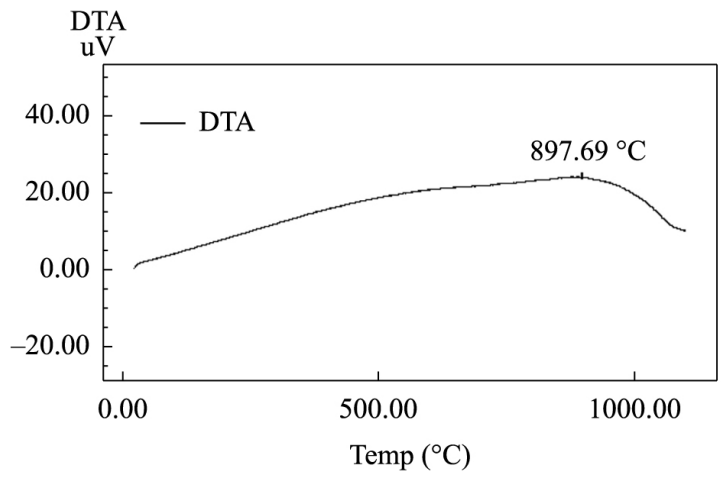

Figure 1. Differential thermal analysis (DTA) curve for uncoated diamonds.

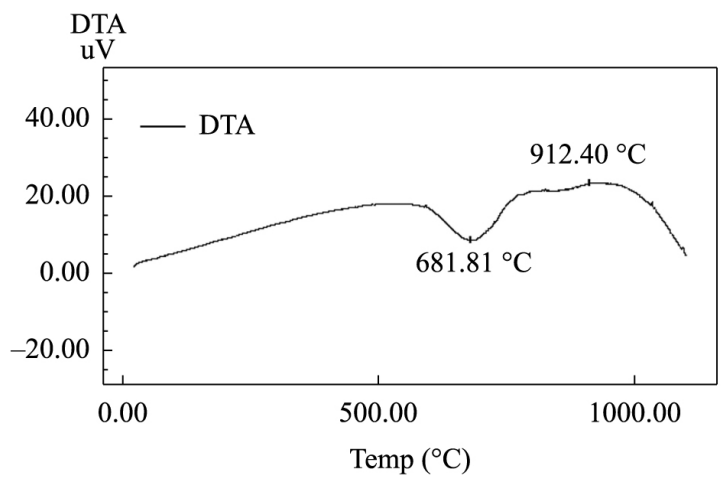

Figure 2. Differential thermal analysis (DTA) curve for Ti-coated diamonds.

(Figure 5a). For Ti-coated diamonds (Figure 5b), it was verified a higher composition of titanium, as expected.

\subsubsection{After heat treatment during $60 \mathrm{~min}$}

Analyzing the diffractograms for the uncoated diamonds (Figure 6a), it is noticed that the two peaks related to the diamond phases were maintained. However, the peaks show lower intensities, and the background is noisier, characterizing the presence of amorphous phases, possibly amorphous carbon (beginning of the graphitization). In relation to the Ti-coated diamonds (Figure 6b), it can be noted the appearance of two peaks with angle $2 \theta$ of $27.44^{\circ}$ and $54.50^{\circ}$. These peaks are inherent to titanium oxide $\left(\mathrm{TiO}_{2}\right)$, from the performing of heat treatment.

Observing the micrograph of the uncoated diamonds in Figure 7a, it can be seen great evidences of thermal degradation, in example, craters on the side. The triangles that take place on the surface of the diamond characterize graphitization sites ${ }^{5,10-12}$. Analyzing the micrograph of the Ti-coated diamonds (Figure 7b), it is possible to observe that the superficial layer presents cracks. This layer can be related to the titanium oxide.

As the diffractogram of the Ti-coated diamonds does not show a noisy background, it is supposed that in this case the formation of amorphous phase did not take place, reinforcing the protective effect of the oxide layer formed on the diamond surface. 


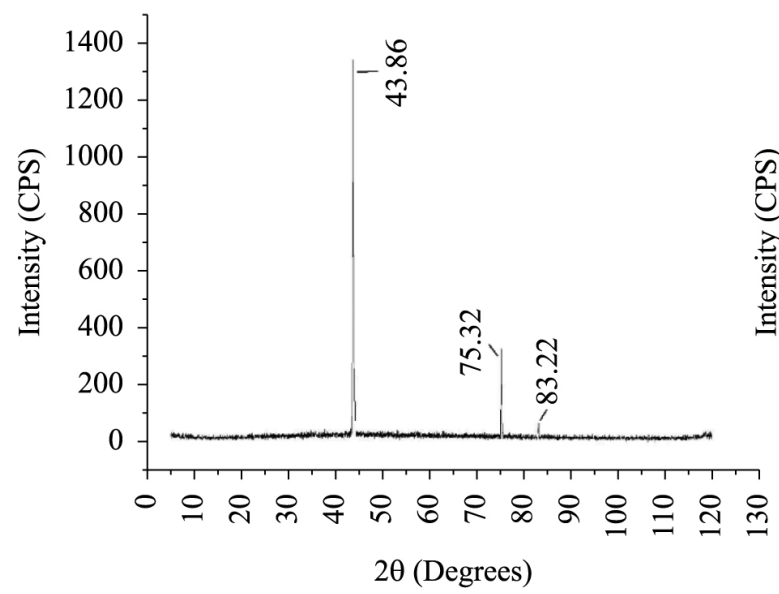

(a)

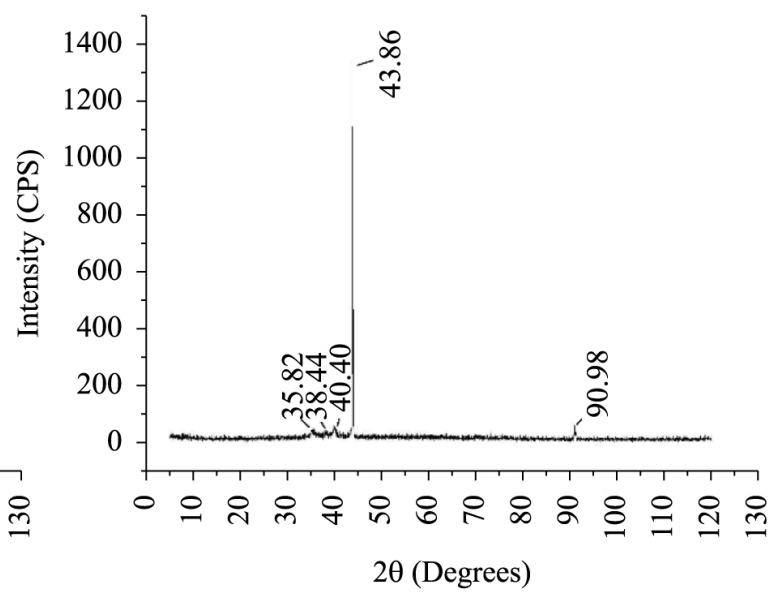

(b)

Figure 3. Diffractogram of (a) uncoated diamonds and (b) Ti-coated diamonds, prior to heat treatment.

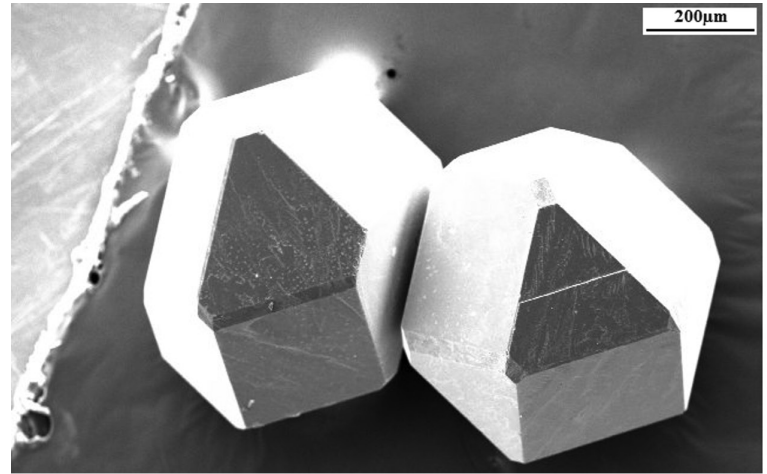

(a)

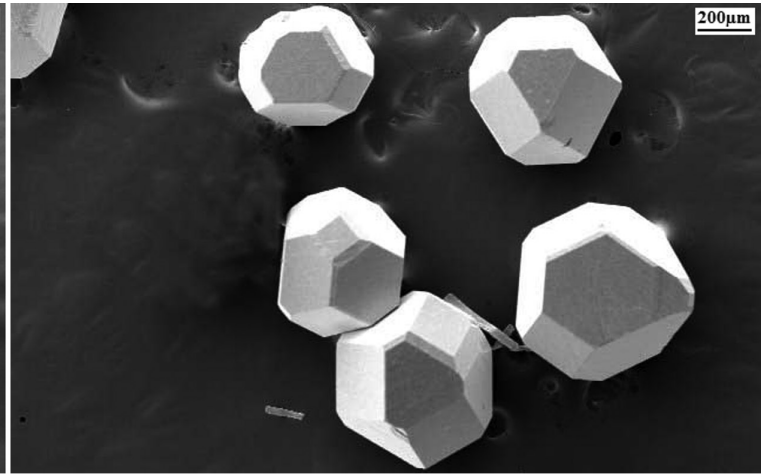

(b)

Figure 4. Diamonds (a) uncoated and (b) with Ti coating, prior to heat treatment.

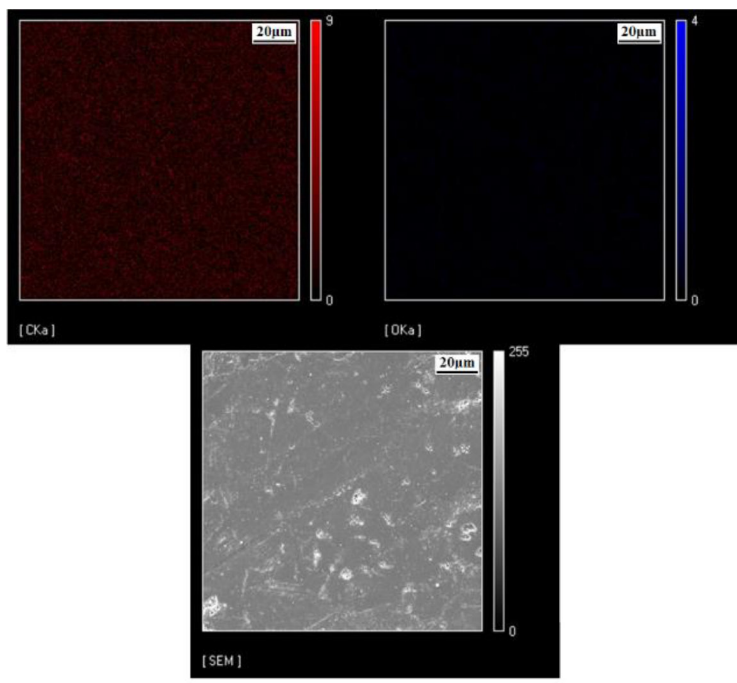

(a)

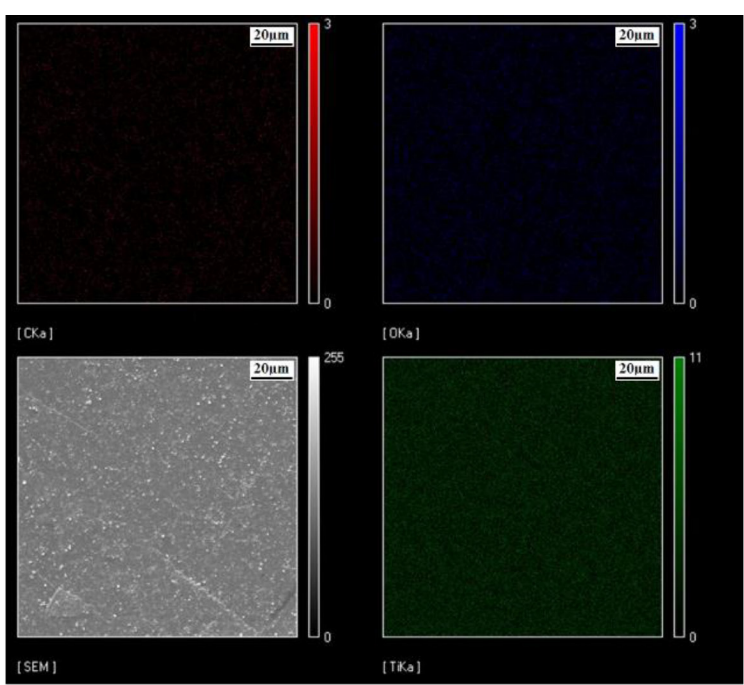

(b)

Figure 5. Mapping by EDS for (a) uncoated diamonds and for (b) Ti-coated diamonds, prior to heat treatment. 


\subsubsection{After heat treatment during $180 \mathrm{~min}$}

Analyzing the diffractogram of the uncoated diamonds (Figure 8a), it can be observed a background more noisy and peaks with lower intensities than diamonds treated during
60 min, which indicates that the amount of amorphous phase increases with the increasing time of heat treatment. A new peak of high intensity appeared with angle $2 \theta$ of $50.60^{\circ}$ associated to the formation of carbon-rich phases. The peak

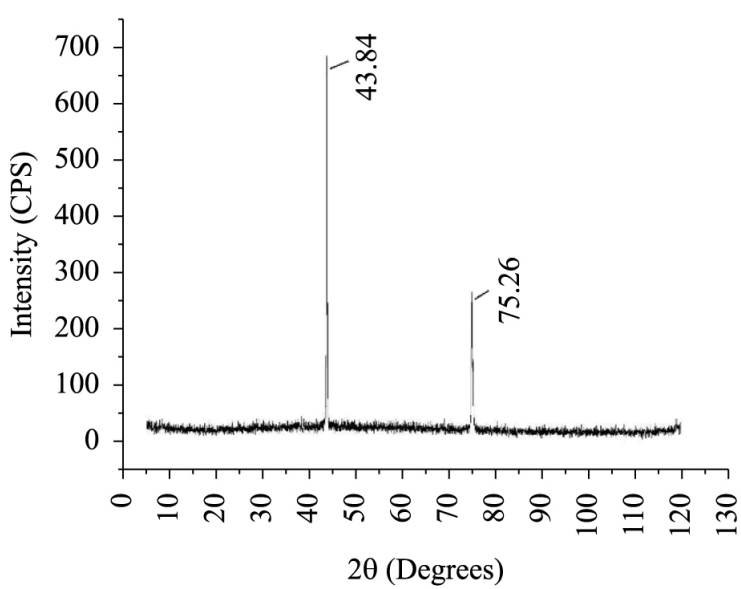

(a)

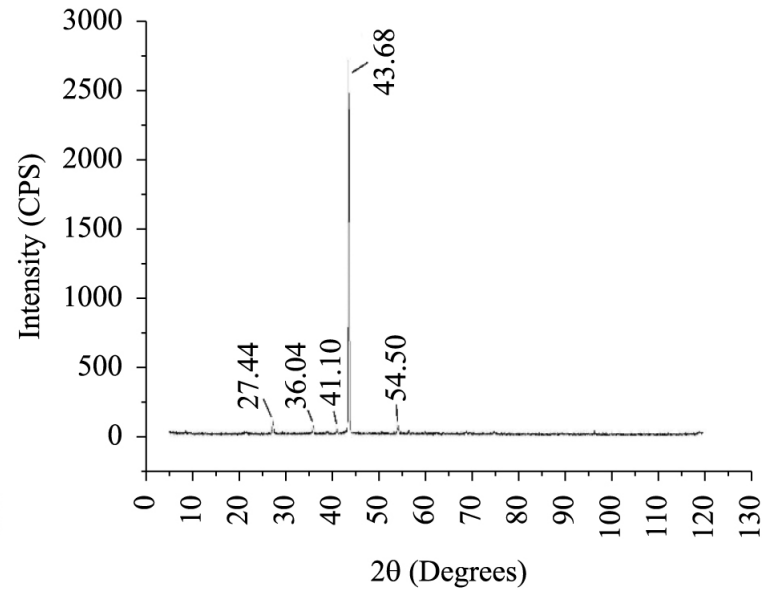

(b)

Figure 6. Diffractogram of (a) uncoated diamonds and (b) Ti-coated diamonds, after heat treatment during $60 \mathrm{~min}$.

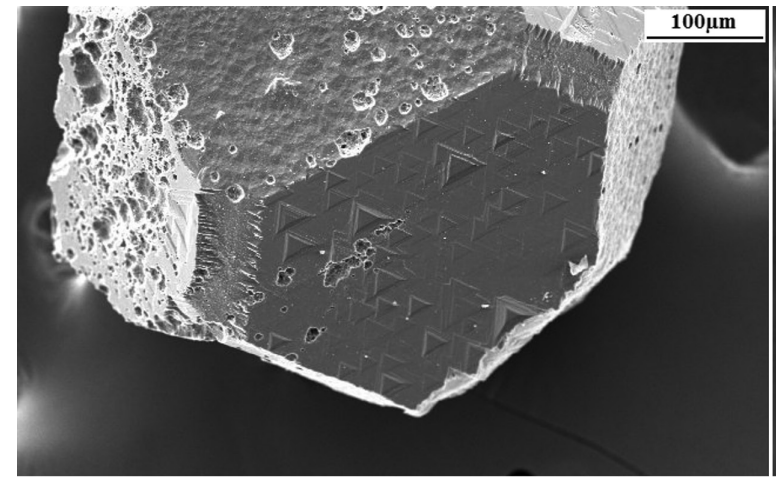

(a)

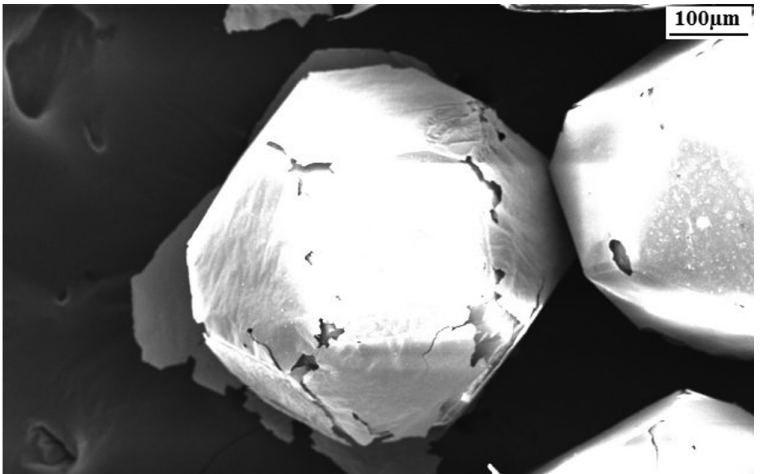

(b)

Figure 7. Diamonds (a) without coating and (b) with Ti coating, after heat treatment during $60 \mathrm{~min}$.

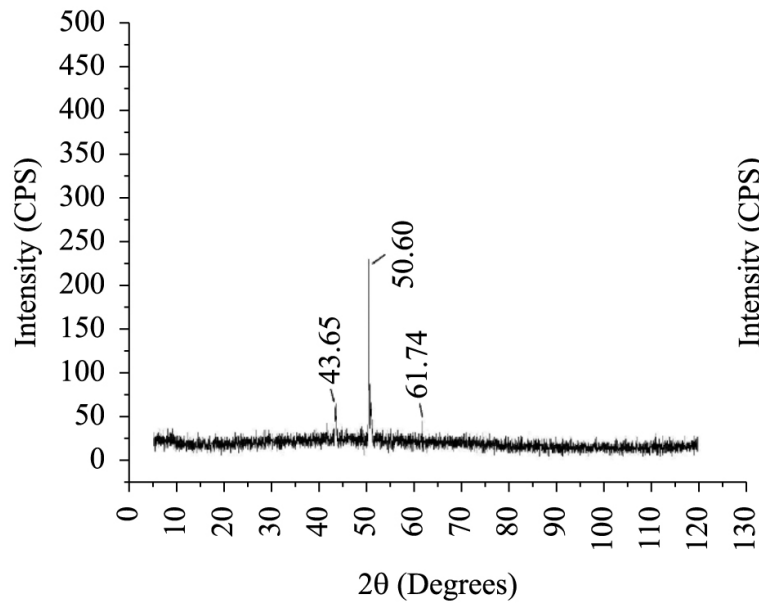

(a)

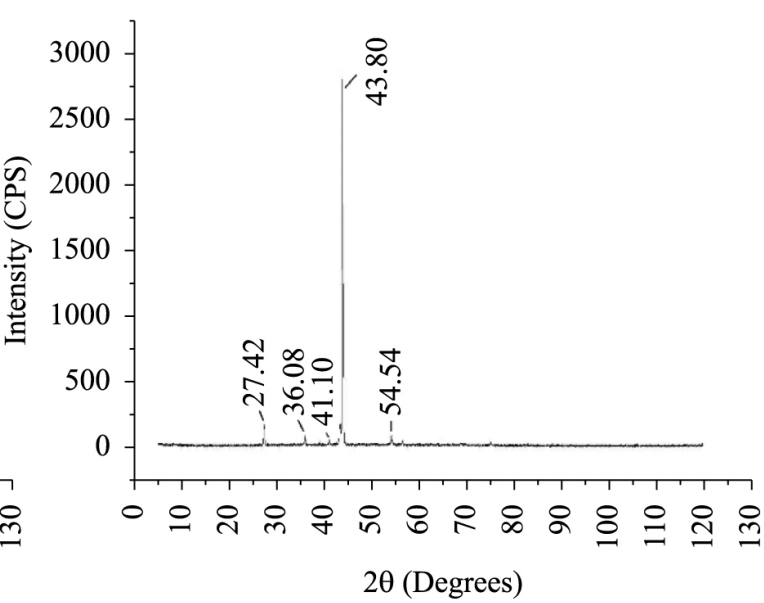

(b)

Figure 8. Diffractogram of (a) uncoated diamonds and (b) Ti-coated diamonds, after heat treatment during $180 \mathrm{~min}$. 
with angle $2 \theta$ of $61.74^{\circ}$ has a very thin base, very low intensity and was not found in the database.

The diffractogram of Ti-coated diamonds (Figure 8b) shows only changes in the intensities of the peaks, related to the formed oxides and small changes in the position of all peaks. It is clear the increase of the intensities of titanium oxide peaks, mainly the peak with angle $2 \theta$ of $27.42^{\circ}$. It suggests that with the increase of treatment time, more titanium oxide was formed.

Analyzing the uncoated diamonds in Figure 9a, it can be noticed that graphitization sites are not found due to the increase in the amount of amorphous phase, as reported in the diffractogram from Figure 8a. It is remarkable the presence of craters on the diamond surface, that were probably originated from triangular sites. In the micrograph of the Ti-coated diamonds (Figure 9b), the oxide layer starts to be detached, exposing the surface of the diamond. This surface shows fewer defects than the surface of the uncoated diamonds, heat treated during $60 \mathrm{~min}$. - see Figure $7 \mathrm{a}$, due to the protection that the layer provided.
Analyzing the mapping by EDS for the uncoated diamonds (Figure 10a), it is noticed that the content of carbon increases due to the formation of amorphous phases, and that oxygen increases due to the oxidation of diamonds. By the mapping of the Ti-coated diamonds (Figure 10b), it can be observed the detachment of the layer of titanium oxide. The exposed surface of the diamond is mostly composed of carbon, and the region inherent to the protective layer is composed of oxygen and titanium. It is interesting to notice that in Figure $10 \mathrm{~b}-$ the spectrum of $\mathrm{Ti}$, the upper region shows few, or no titanium, and the lower half is rich in Ti. It justifies the fact that the lower portion is plain and free of defects (diamond face that remained coated), and the upper half shows thermal damage (craters) - due to some graphitization.

\subsubsection{After heat treatment during $360 \mathrm{~min}$}

The diffractogram of the uncoated diamonds (Figure 11a) shows a background totally noisy. It indicates that the depth detected by X-ray diffraction corresponds only to the amorphous phase. For this reason, diamonds' surfaces are totally covered by amorphous carbon. Analyzing the

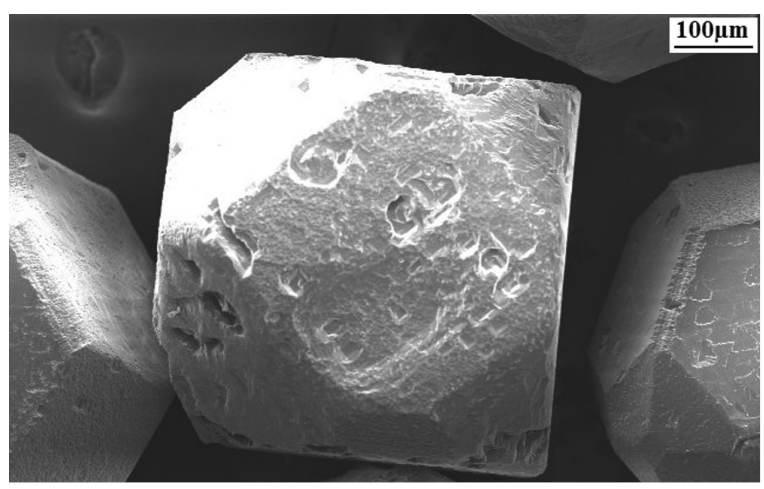

(a)

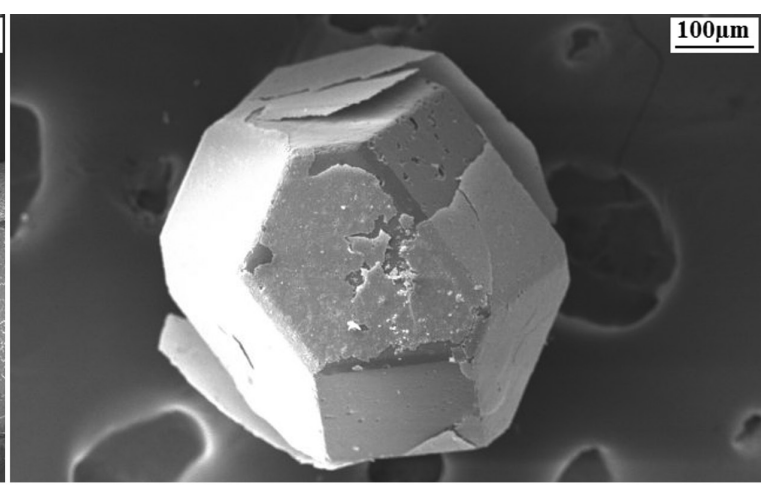

(b)

Figure 9. Diamonds (a) without coating and (b) with Ti coating, after heat treatment during $180 \mathrm{~min}$.

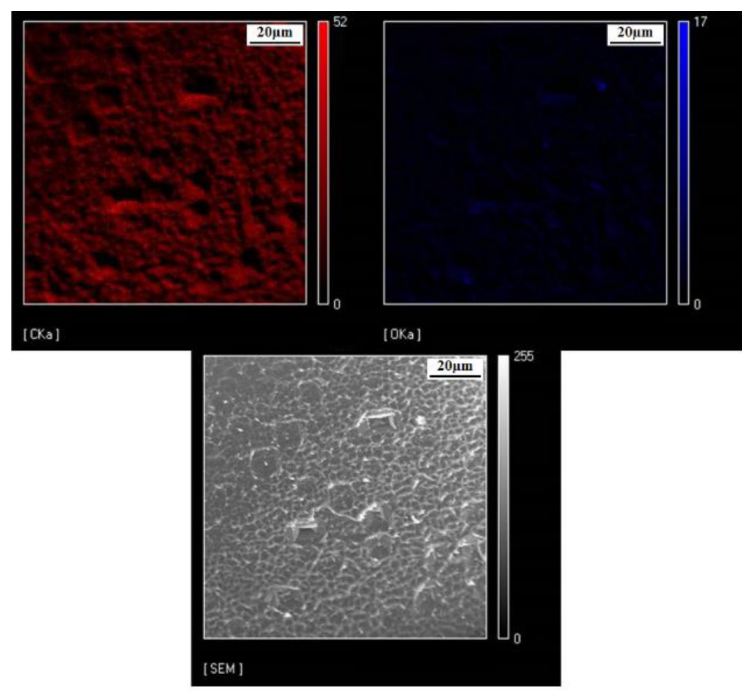

(a)

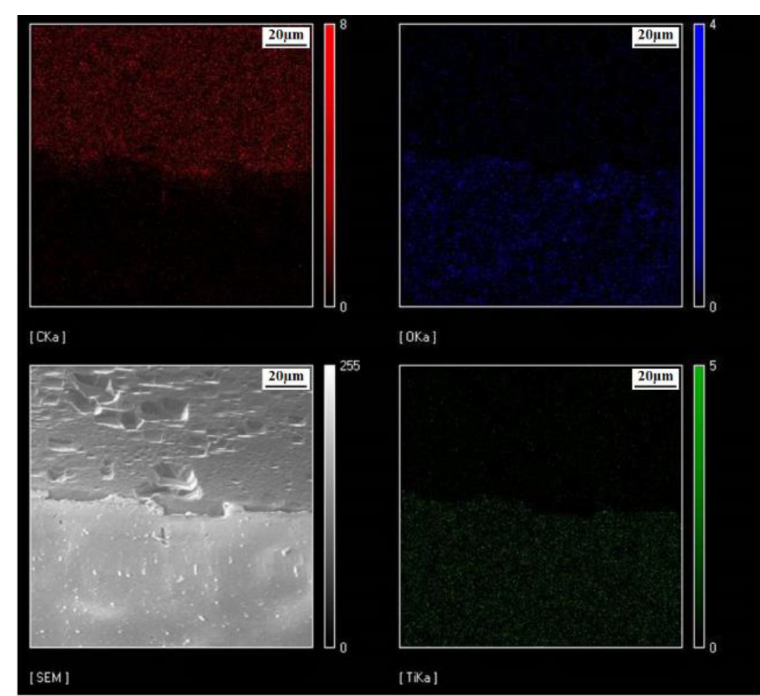

(b)

Figure 10. Mapping by EDS for (a) uncoated diamonds and for (b) Ti-coated diamonds, after heat treatment during $180 \mathrm{~min}$. 


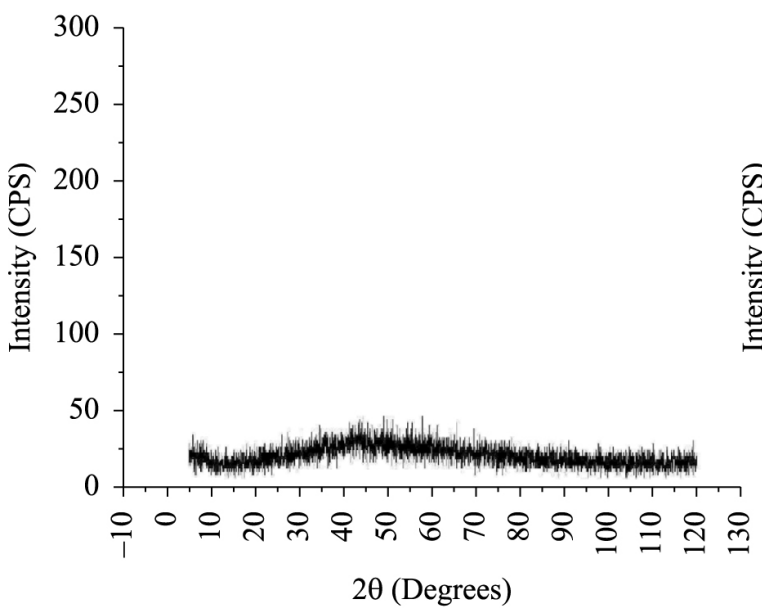

(a)

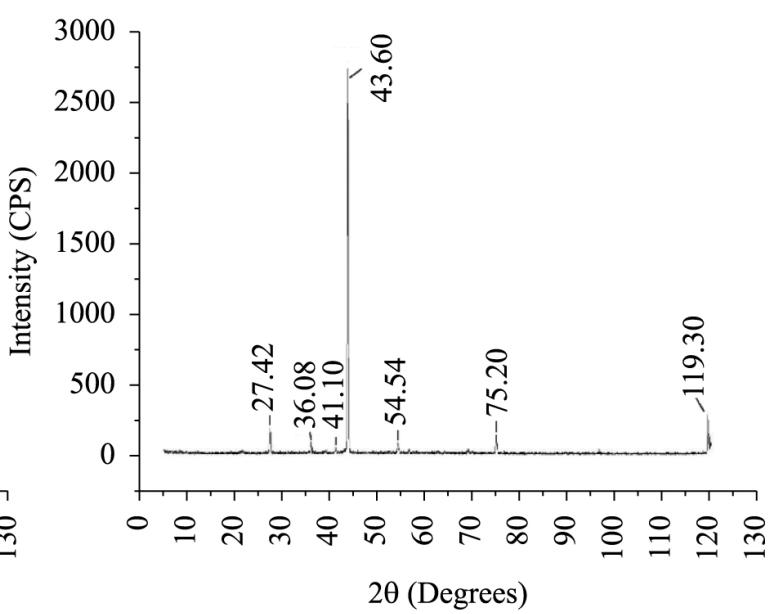

(b)

Figure 11. Diffractogram of (a) uncoated diamonds and (b) Ti-coated diamonds, after heat treatment during 360 min.

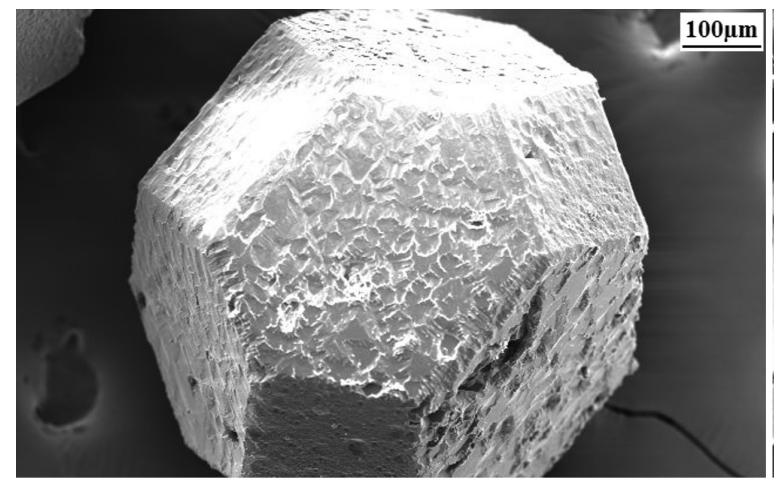

(a)

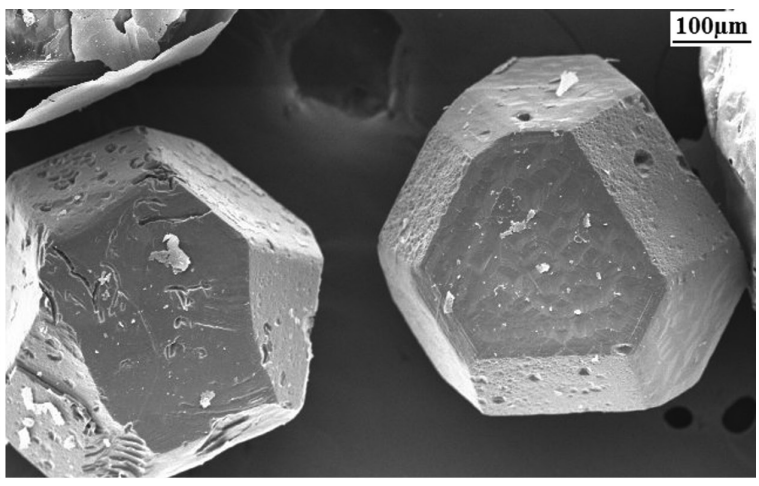

(b)

Figure 12. Diamonds (a) without covering and (b) with Ti coating, after heat treatment of $360 \mathrm{~min}$.

diffractogram of the Ti-coated diamonds (Figure 11b), it is noticed the appearance of two new peaks, with angle $2 \theta$ of $75.20^{\circ}$ and $119.30^{\circ}$. The first peak is inherent to diamond and the second to metallic Ti. With the increasing of heat treatment time, much of the titanium oxide coating was removed, thus exposing the diamond surface - explaining $2 \theta=75.20^{\circ}$. The peak at $2 \theta=119.30^{\circ}$ indicates that oxigen did not diffused to the diamonds' surfaces, even for longer times (360 min.), once a metallic Ti coat was found yet, under the titanium oxide coating.

Figure 12a clearly shows that the diamond crystal is completely covered by a layer with texture different from the original - as received - see Figure 4a, which according to the amorphous character obtained by X-ray diffraction, is a layer of amorphous carbon. Figure $12 \mathrm{~b}$ shows that for Ti-coated diamonds, the oxide layer was removed almost completely. The exposed diamonds are in good conditions, presenting only few defects on the surface.

It is important to point that, in general, the DTA results are in close agreement with the analyses carried out by XRD and EDS. Extensive graphitization of the uncoated diamond crystals has been observed. Meanwhile, the Ti-coated diamond crystals show the following: (1) initial oxidation of the Ti coating, followed by (2) the break and detachment of the $\mathrm{TiO}_{2}$ coating, culminating with (3) their slight graphitization. In this sense, the Ti coating showed remarkable protective effect against the thermal damage in diamonds, even during prolonged periods.

\section{Conclusions}

The main conclusions drawn from this work are as follows:

(i) Two mechanisms of degradation of the diamonds have been observed: oxidation and graphitization;

(ii) Analyzing the heat-treated microstructure of uncoated diamonds, it was possible to observe graphitization sites. However, as long as the treatment time increases, the diamond surface is covered by a layer of amorphous carbon (graphitization), evidenced by the noisy background in the diffractograms;

(iii) For Ti-coated diamonds, a layer of titanium oxide was formed, as indicated in the diffractograms. This layer has a protective character; 
(iv) As long as the treatment time increases, the $\mathrm{TiO}_{2}$ detaches itself, exposing the diamond surface;

(v) It was found that after 360 minutes of heat treatment, the surface of Ti-coated diamonds did not show many defects in relation to the uncoated diamonds, and this latter was totally covered by a layer of amorphous carbon;

\section{References}

1. Davis PR, Fish ML, Peacock S and Wright DN. An indicator system for saw grit. Industrial Diamond Review. 1996; 56(57):7887.

2. Moreira V Jr. Processing and characterization of diamond wires'beads. [Dissertation]. Rio de Janeiro: State University of North Fluminense; 2013. 166 p. (in portuguese).

3. Tankala K, Debroy T and Alam M. Oxidation of diamond films syntherized by hot filament assister chequical vapor deposition. Journal of Materials Research. 1990; 5(11):2483-2489. http:// dx.doi.org/10.1557/JMR.1990.2483.

4. Cabral SC. Influence of the use of coatings on diamonds on the properties of diamond composites obtained by powder metallurgy. [Dissertation]. Rio de Janeiro: State University of North Fluminense; 2009. 107 p. (in portuguese).

5. Oliveira HCP. Influence of niobium additions to the iron-copper system for use as metal matrix in diamond cutting tools. [Thesis]. Rio de Janeiro: State University of North Fluminense; 2010. 205 p. (in portuguese).

6. Howe JY, Joles LE and Cormack AN. The oxidation of diamond. Alfred: School of Ceramic Engineering and Materials Science/ Alfred University; 2001. p. 1-5. (vi) It has been clearly proven in this paper that the Ti coating provides a longer life for diamonds, indicating potential benefits for diamond tools.

\section{Acknowledgements}

The authors would like to express their gratitude to CNPq and Element Six (e6).

7. John P, Polwarta N, Troupe CE and Wilson JIB. The oxidation of (100) textured diamond. Diamond and Related Materials. 2002; 11(3-6):861-866. http://dx.doi.org/10.1016/S09259635(01)00673-2.

8. Lee JK, Michael W, Anderson F, Gray A and John P. Explosive oxidation of HPHT diamond particles. Diamond and Related Materials. 2006; 51(8):1206-1209. http://dx.doi.org/10.1016/j. diamond.2005.06.033.

9. Sun CQ, Xie H, Zhang W, Ye H and Hing P. Preferential oxidation of diamond $\{111\}$. Journal of Physics. D, Applied Physics. 2000; 33(17):2196-2199. http://dx.doi.org/10.1088/00223727/33/17/316.

10. Evans $\mathrm{T}$ and Phaal $\mathrm{C}$. The kinetics of the diamond: oxygen reaction. In: Proceedings of the 5th Conference on Carbon; Pennsylvania; 1962. New York: Pergamon Press; 1962. p. 147153.. http://dx.doi.org/10.1016/B978-0-08-009707-7.50025-X.

11. Rosa MCG. Thermal damage in diamonds. [Dissertation]. Rio de Janeiro: State University of North Fluminense; 2011.91 p. (in portuguese).

12. Chagas VM. Influence of a TiC coating on the thermal resistance of diamonds. [Dissertation]. Rio de Janeiro: State University of North Fluminense; 2012. 124 p. (in portuguese).

13. Evans T. The properties of diamonds. London: Academic Press; 1979. v. 18 , n. 2, p. 403-424. 